\title{
Gastroenteropancreatic neuroendocrine tumor metastases to the thyroid gland: differential diagnosis with medullary thyroid carcinoma
}

\author{
S Leboulleux, E Baudin, J Young ${ }^{1}$, B Caillou ${ }^{2}$, V Lazar $^{3}$, G Pellegriti, M Ducreux ${ }^{4}$, G Schaison ${ }^{1}$ and \\ M Schlumberger \\ Service de Médecine Nucléaire, Institut Gustave-Roussy, Villejuif, France, ${ }^{1}$ Service d'Endocrinologie, Hôpital de Kremlin Bicêtre, France, ${ }^{2}$ Département \\ de Pathologie, Institut Gustave-Roussy, Villejuif, France, ${ }^{3}$ Département de Biologie Clinique, Institut Gustave-Roussy, Villejuif, France, ${ }^{4}$ Département de \\ Médecine, Institut Gustave-Roussy, Villejuif, France \\ (Correspondence should be addressed to E Baudin, Institut Gustave-Roussy, 39 rue Camille Desmoulins, 94805 Villejuif Cedex, France)
}

\begin{abstract}
Neuroendocrine tumors (NET) of the thyroid gland are rare. Apart from medullary thyroid carcinoma (MTC), metastases of gastroenteropancreatic (GEP) NET may also occur. Features of six patients (five men, one female; age range, 39-67 years) with thyroid metastases from a GEP-NET are described. Thyroid metastases were bilateral in all patients and were associated with enlarged neck lymph nodes in five. In four cases, the thyroid tumor was either the first sign of the disease $(n=2)$ or was an isolated site of recurrence $(n=2)$. The tumors were well $(n=3)$ or poorly differentiated $(n=3)$. Five tumors for which the primary site could be determined corresponded to foregut-derived tumors (3 lungs, 1 thymus and 1 pancreatic NET). One tumor demonstrated calcitonin (CT) production as shown by immunohistochemistry and elevated plasma CT levels. However, the disease history and the clinical features strongly favored a metastasizing GEP-NET. No tumoral RET proto-oncogene mutation was found in this patient. The differential diagnosis between metastatic GEP-NET and MTC is crucial because prognosis, work-up, and treatment differ greatly.
\end{abstract}

European Journal of Endocrinology 140 187-191

\section{Introduction}

Thyroid neuroendocrine tumors (NET) are rare. The most frequent NET tumor arising in the thyroid gland is medullary thyroid carcinoma (MTC) which is derived from C-cells and characterized by calcitonin (CT) production. A familial history is present in $30 \%$ of MTC patients, the consequence of a point mutation in the RET gene, and it may be part of a multiple endocrine neoplasia type 2 in which hyperparathyroidism and pheochromocytoma are known to occur. In their sporadic form, patients typically present with a thyroid tumor, frequently associated with enlarged neck lymph nodes. The only curative treatment is total thyroidectomy and bilateral lymph node dissection. However, only one third of the patients are cured after surgery and the others have detectable CT levels post-surgery that need further treatment. Apart from MTC, metastases from both gastroenteropancreatic neuroendocrine tumors (GEP-NET) and primary paraganglioma can be found in the thyroid gland. Thyroid paraganglioma is exceptional (1), with immunohistochemistry negative for epithelial markers, but positive for neuronal markers. The differential diagnosis of MTC from GEPNET metastasis in the thyroid gland is a crucial challenge for the clinician with major management and therapeutic consequences. The discovery of thyroid metastases indeed modifies the staging of these tumors; chemosensitivity in well differentiated pancreatic tumors and poorly differentiated GEP-NET is much greater than that seen in MTC. However, both share some identical tumor markers such as CT, which is produced by all MTCs and by about $14 \%$ of foregut-derived GEP-NET $(2,3)$. When present, it may be confusing, especially in cases of thyroid metastases.

In the present study we report the clinical, biological and pathological features of six GEP-NET patients with metastases in the thyroid gland.

\section{Materials and methods}

\section{Patients}

Five patients (patients $1-5$, Table 1 ) are part of a series of 130 patients with sporadic GEP-NET in whom a prospective biological screening was performed (3); one recent patient (patient 6) belongs to a multiple endocrine neoplasia type 1 family (Table 1 ). 


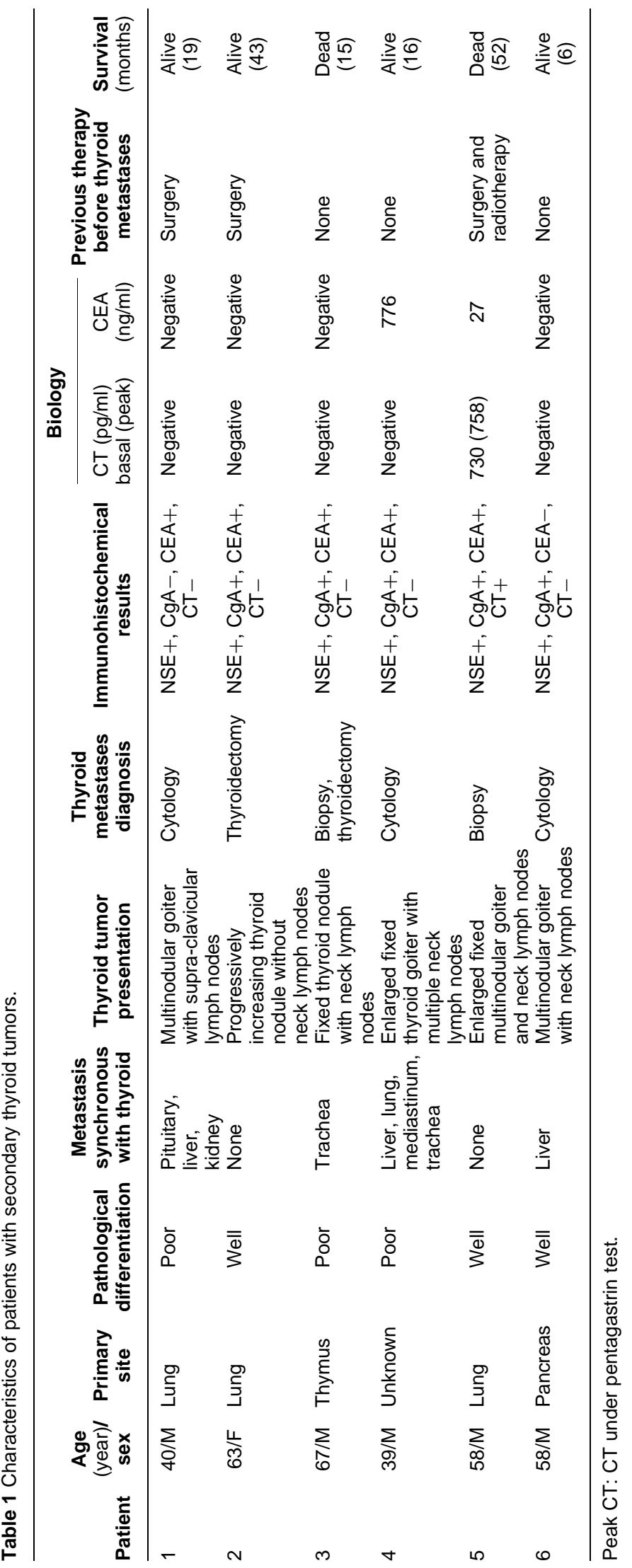




\section{Methods}

Biology In each case, plasma CT levels (ELSA-CT, CisBio International, Gif-sur-Yvette, France; normal value $<10 \mathrm{pg} / \mathrm{l})$ were determined as well as serum carcinoembryonic antigen (CEA) levels (Enzymum-test CEA, Boehringer, Mannheim, Germany; normal value $<7 \mathrm{ng} / \mathrm{ml}$ ). If increased CT levels were found, CT was regularly re-evaluated.

Pathology All pathological specimens were reviewed. Antibodies against neuron-specific enolase (NSE) (DAKO, Giostsup, Denmark), chromogranin A (CgA) (DAKO, Denmark), synaptophysin, (DAKO, Denmark), CT (DAKO, Carpinteria, USA) and CEA (DAKO, Denmark) were used for immunochemistry. Immunostaining with thyroglobulin (DAKO, Denmark) was negative in all tumors. Pathological differentiation was evaluated according to the Warren and Gould classification (4).

Proto-oncogene RET In one patient demonstrating CT secretion (patient 5), screening for RET mutations in exons $10,11,13,14,15$ and 16 was performed by single-strand conformation polymorphism (SSCP) analysis on peripheral blood mononuclear cells and tumoral DNA, as previously described (5).

\section{Results}

The characteristics of the patients are shown in Table 1.

\section{Patient 1}

A 40-year-old man underwent surgery for a 5-cm bronchial tumor. The pathological examination revealed a poorly differentiated neuroendocrine carcinoma. At immunohistochemistry, tumor cells were strongly positive for NSE and negative for $\mathrm{CgA}$. There were no metastatic mediastinal lymph nodes, and the initial work-up did not find any metastasis. Serum levels of NSE and somatostatin were increased. Four months later, a widespread metastatic disease affecting the pituitary, lungs, liver, kidneys and also the thyroid was found. The thyroid presented as a multinodular goiter and thyroid cytology found cells which had the same features as the initial bronchial tumor. The patient was then treated with six courses of chemotherapy associating cisplatin and etoposide. Tumor response was complete, with a normalization of the tumor markers. One year later, the patient is still in complete remission.

\section{Patient 2}

A 63-year-old woman underwent surgery for a 3.5-cm bronchial tumor with mediastinal metastatic lymph nodes. The pathological features were those of a well differentiated neuroendocrine carcinoma. At immunohistochemistry, tumor cells were strongly positive for NSE, synaptophysin, CgA and CEA. They were negative for CT. The only circulating tumor marker was $\mathrm{CgA}$. After surgery, the only abnormality was a $1-\mathrm{cm}$ thyroid nodule. A fine needle aspiration showed neoplastic cells that led to a total thyroidectomy with cervical lymph node dissection. Pathological examination found multiple and bilateral thyroid metastases that had the same features as the bronchial NET. There was no metastatic lymph node. Four months later, a disseminated disease affecting adrenals and liver was found. The patient was then treated with somatostatin analogs, but disease progression led to chemotherapy associating 5-fluorouracil and streptozocin, that is currently in progress.

\section{Patient 3}

A 67-year-old man with a history of Graves' disease presented with a large and fixed thyroid tumor and enlarged neck lymph nodes. A thyroid tumor biopsy led to the diagnosis of a poorly differentiated NET. At that time, no primary site was found and there was no increase in circulating tumor markers. The patient was treated with six courses of a combination of cisplatin and etoposide, leading to a partial response. A total thyroidectomy with lateral tracheal resection and lymph node dissection was then performed. Pathological examination found a poorly differentiated neuroendocrine carcinoma with the presence of Hassall's bodies. These features suggested that the primary tumor was a poorly differentiated thymoma with a massive loco-regional involvement. The tumor cells were positive for NSE, CgA and CEA and negative for CT. Two months after surgery, multiple bone metastases were discovered and the patient died a few weeks later.

\section{Patient 4}

A 39-year-old man presented with a fixed goiter with enlarged lymph nodes in the neck and mediastinum, and liver and pulmonary metastases. Thyroid cytology showed neoplastic cells and neck lymph node biopsy revealed a poorly differentiated neuroendocrine carcinoma; the tumor cells were positive for NSE, $\mathrm{CgA}$ and CEA and negative for CT. No primary site was found. Serum CEA and NSE levels were increased but plasma CT level was normal. The patient was treated with 6 courses of chemotherapy associating cisplatin and etoposide leading to a major tumor response with a normalization of serum markers. Brain metastases appeared 4 months after chemotherapy had been discontinued and brain radiotherapy is currently in progress.

\section{Patient 5}

A 58-year-old man with a long history of goiter had lung surgery for a $15-\mathrm{cm}$ bronchial tumor with involvement of the proximal lymph nodes. Circulating CT, CEA, NSE and glycoprotein human alpha subunit 
levels were increased. CT level did not increase following pentagastrin stimulation. Histology diagnosed a well differentiated NET indistinguishable from MTC with tumor cells positive for CEA, CT, CgA and keratin. No other tumoral localization was found and mediastinal radiotherapy was performed. Two years later, the patient presented with a rapid enlargement of the thyroid gland, multiple enlarged lymph nodes in the neck, laryngeal nerve palsy and dysphagia. Neck surgery was undertaken. Thyroid tumor and neck lymph nodes shared the same pathological and immunohistochemical features as the initial bronchial tumor. Chemotherapy associating 5-fluoro-uracil, dacarbazine, doxorubicin and streptozocin was administered. A major tumor response was observed with normalization of the circulating markers, but this lasted only three months, after which death occurred rapidly. Neither germinal nor somatic RET gene mutation was found.

\section{Patient 6}

A 58-year-old man with multiple bone metastases had thyroid nodules with enlarged neck lymph nodes. The abdominal scan and ultrasonography showed a 3-cm pancreatic tumor. The diagnosis of a pancreatic NET with thyroid and bone metastases was raised and familial screening revealed a multiple endocrine neoplasia type 1. A neck lymph node biopsy showed a well differentiated NET. The thyroid cytology confirmed the diagnosis of thyroid metastasis. Circulating NSE and CgA levels were elevated, but CT levels were normal. Chemotherapy associating doxorubicin and streptozocin is currently in progress.

\section{Discussion}

Metastatic tumors to the thyroid gland provide the clinician and the pathologist with a diagnostic challenge, especially in patients without previous medical history of cancer and when thyroid metastasis is isolated $(6,7)$. In these cases, previous studies have demonstrated the poor efficiency of cytological examination to differentiate a metastasis to the thyroid from a primary thyroid carcinoma and also the possibility of misinterpretation of the final histology. This problem may be even more obvious in differentiating GEP-NET metastases from primary thyroid NET. MTC is the most frequent thyroid NET. Its diagnosis is based on morphological examination and is confirmed by the demonstration of CT production, as shown by plasma measurement and immunohistochemistry. In contrast, normal circulating CT levels and negative immunohistochemistry with CT antibodies classically rule out the diagnosis of MTC in patients with a clinical thyroid tumor. However, the production of CT is not specific for MTC, and its level can be increased in other tumors, mainly GEP-NET $(2,8-11)$. Other NET may also be found in the thyroid gland, mainly GEP-NET metastasis, as emphasized in this study.

Our study demonstrates a 4\% (5 of 130) frequency of thyroid metastases in patients with GEP-NET. Some general remarks concerning our patients should be made: (i) all patients but one (patient 2) had a thyroid tumor that was clinically evident and suspicious for neoplastic disease; (ii) in 4 patients, the thyroid tumor was either the first sign of the disease (patients 3 and 4) or an isolated site of recurrence (patients 2 and 5); (iii) tumors disclosed various degrees of differentiation; (iv) all patients with a known primary had a foregut-derived NET. In patients with thyroid NET, normal plasma levels of CT and negative immunohistochemistry with CT antibodies rule out MTC diagnosis, and this was the case in five patients (patients 1-4 and 6). Among these five patients, three (patients 1,2 and 5) had a previous history of NET and cytological and pathological features of the thyroid tumor were similar to those of the initial NET. Of note, the cytological diagnosis of thyroid NET metastases in three out of six patients (patients 1,2 and 5) was made in all cases by comparing cytological results with previous pathological data. Nevertheless, in all three cases the diagnosis of thyroid neoplasia was made by cytology. Furthermore, it should be underlined that in three patients (patients 1, 3 and 4) with a poorly differentiated tumor, the neuroendocrine features were shown by immunohistochemistry with general neuroendocrine markers (NSE, CgA, synaptophysin). Furthermore, three of them experienced a more rapid progression of the thyroid tumor than is usually seen in MTC.

In patients with a thyroid NET and CT production as shown by elevated circulating CT levels and immunohistochemistry, both an MTC and a GEP-NET metastasis to the thyroid gland should be considered. This was the case in one patient (patient 5). This patient with a bronchial NET had a recurrence in the thyroid and neck lymph nodes two years after initial diagnosis. We recently reported a $14 \%$ incidence of CT secretion in patients presenting with GEP-NET (3). Patient history, clinical presentation, hormone measurements including a pentagastrin test, and a search for RET mutation may help to differentiate an MTC from a GEP-NET thyroid metastasis. Our patient had a known GEP-NET primary and the thyroid tumor was found to be a secondary event. Furthermore, CT did not increase following pentagastrin stimulation, suggesting that the thyroid tumor was not an MTC. This test is reported to discriminate between patients with MTC demonstrating an explosive response of CT, and other NET. It should be noted, however, that no results of the pentagastrin stimulation test in MTC patients with high basal CT levels have yet been reported. We have reported a $19 \%$ prevalence of the secretion of glycoprotein human alpha subunit in GEP-NET patients, mainly of foregut origin (3); this has also been found in rare MTC patients (12), but was absent in 19 MTC patients studied in our 
department (L Guignat \& E Baudin, unpublished data). Therefore, when the level of glycoprotein human alpha subunit is increased, this may help to distinguish MTC from GEP-NET thyroid gland metastasis. CEA is a classical MTC marker but its increase has been reported in various cancers. Somatic mutations of the RET protooncogene have been reported in $15-65 \%$ of sporadic MTC (13-15), in a few sporadic pheochromocytomas $(16,17)$, and among the other NET, only in small cell lung carcinomas $(17,18)$. Therefore, a somatic RET proto-oncogene mutation favors the diagnosis of MTC, but its absence, as in our patient, is not conclusive.

In conclusion, our study highlights the incidence of thyroid gland metastasis in patients with GEP-NET. In patients with a known NET, or with a poorly differentiated thyroid tumor, even if there is CT secretion, the final diagnosis of a GEP-NET thyroid metatasis should be raised. Consequences on patient work-up and therapeutic options justify this strategy.

\section{References}

1 LaGuette J, Matias-Guiu X \& Rosai J. Thyroid paraganglioma: a clinicopathologic and immunohistochemical study of three cases. American Journal of Surgical Pathology 199721 748-753.

2 Ghillani PP, Motté P, Troalen F, Julienne A, Gardet P, Le Chevalier $\mathrm{T}$ et al. Identification and measurements of calcitonin precursors in serum of patients with malignant diseases. Cancer Research $1989496845-6851$.

3 Baudin E, Bidart JM, Rougier P, Lazar V, Ruffié P, Ropers J et al. Screening for multiple endocrine neoplasia type 1 and hormona production in apparently sporadic neuroendocrine tumors. Journal of Clinical Endocrinology and Metabolism 199984 69-75.

4 Warren WH, Gould VE, Faber LP, Kittle FC \& Memoli VA. Neuroendocrine neoplasms of the bronchopulmonary tract. Journal of Thoracic and Cardiovascular Surgery $198589819-825$.

5 Lazar V, Grandjouan S, Bognel C, Couturier D, Rougier P, Bellet D et al. Accumulation of multiple mutations in tumour suppressor genes during colorectal tumorigenesis in HNPCC patients. Human Molecular Genetics 199412 2257-2260.

6 Matias-Guiu X, LaGuette J, Puras-Gil AM \& Rosai J. Metastatic neuroendocrine tumors to the thyroid gland mimicking medullary carcinoma: a pathologic and immunohistochemical study of six cases. American Journal of Surgical Pathology 199721 754762 .
7 Nakhjavani MK, Gharib H, Goellner JR \& Van Heerden JA. Metastasis to the thyroid gland: a report of 43 cases. Cancer 1997 73 574-578.

8 Vilde F, Arkwright S, Paoli C, Perichon I, Le Charpentier Y \& Le Bodic MF. Neuroendocrine carcinoma of the larynx with secretion of calcitonin: primary tumor or metastasis of the medullary thyroid carcinoma. Annales de Pathologie 199616 104-107.

9 Insabato L, De Rosa G, Terracciano LM, Lupoli G, Montedoro D \& Ravetto C. A calcitonin-producing neuroendocrine tumor of the larynx: a case report. Tumori 1993 79 227-230.

10 Sim SJ, Glassman AB, Ro JY, Lee JJ, Logothetis CJ \& Liu FJ. Serum calcitonin in small cell carcinoma of the prostate. Annals of Clinical and Laboratory Science 199626 487-495.

11 McLeod MK \& Vinik AI. Calcitonin immunoreactivity and hypercalcitoninemia in two patients with sporadic, nonfamilial, gastroenteropancreatic neuroendocrine tumors. Surgery 1992 $111484-488$

12 Nobels F, Kwekkeboom D, Coopmans W, Schoenmakers C, Lindemans J, De Herder W et al. Chromogranin A as serum marker for neuroendocrine neoplasia: comparison with neuronspecific enolase and the $\alpha$ subunit of glycoprotein hormones. Journal of Clinical Endocrinology and Metabolism 199782 2622-2628.

13 Wohllk N, Cote GJ, Bugalho MM, Ordonez N, Evans DB, Goepfert H et al. Relevance of RET proto-oncogene mutations in sporadic medullary thyroid carcinoma. Journal of Clinical Endocrinology and Metabolism 199681 3740-3745.

14 Eng C, Mulligan LM, Smith DP, Healey CS, Frilling A, Raue F et al. Mutation of the RET proto-oncogene in sporadic medullary thyroid carcinoma. Genes, Chromosomes, Cancer 199512 209-212.

15 Zedenius J, Larsson C, Bergholm U, Bovée J, Svensson A, Hallengren B et al. Mutations of codon 918 in the RET protooncogene correlate to poor prognosis in sporadic medullary thyroid carcinomas. Journal of Clinical Endocrinology and Metabolism $1995803088-3090$.

16 Eng C \& Mulligan LM. Mutations of the RET proto-oncogene in the multiple endocrine neoplasia type 2 syndromes, related sporadic tumors, and Hirschsprung disease. Human Mutations 1997 9 97109.

17 Komminoth P, Roth J, Muletta-Feurer S, Saremaslani P, Seelentag WKF \& Heitz PU. RET proto-oncogene point mutations in sporadic neuroendocrine tumors. Journal of Clinical Endocrinology and Metabolism 199681 2041-2046.

18 Futami H, Egawa S, Tsukada T, Maruyama K, Bandoh S, Noguchi $\mathrm{M}$ et al. A novel somatic point mutation of the RET protooncogene in tumor tissues of small cell lung cancer patients. Japanese Journal of Cancer Research 199586 1127-1130.

Received 14 August 1998

Accepted 4 December 1998 